\title{
IOT AND NEURAL NETWORK BASED MULTI REGION AND SIMULTANEOUS LEAKAGE DETECTION IN PIPELINES
}

\author{
Pradyot Aramane, Akshay Bhattad, Madhwesh.M, Nishant Aithal, Akshay.P, \\ Prof.Prapulla S.B and Dr.Shoba.G \\ R. V College of Engineering, Bengaluru-560059 \\ India.
}

\begin{abstract}
The increasing demand for water arising from global population growth and urbanization in recent years is stressing the water supply to its limits. By 2025, 1.8 billion people will experience absolute water scarcity, and 2/3 of the world will be living under water-stressed conditions [1-2]. Neural networks have proved to be an apt approach for water leakage detection as they have the best and most extensive reach on the problem owing to their computational nature. They do not any have basic flaws unlike alternate methods like leakage detection using acoustic sensors which cannot differentiate between spikes in flow and leakage. They are a flexible and efficient approach to detection of leakages in water distribution networks. According to an inquiry made by the International Water Supply Association (IWSA), the amount of lost or "unaccounted for water" (UFW) is typically in the range of 20-30\% of production [3]. In this project, a neural network model is proposed for detection and location of leakages in the pipes based on pressure values from sensors deployed along the pipeline. The network is initially trained using these pressure values and can then be used to detect abnormalities in the readings which can be due to leakages. The open source tool used to develop this model is Neuroph Studio. Neuroph is a neural network framework written in Java. It can be used to create and train neural networks in Java programs. Neuroph provides Java class library as well as GUI tool to quickly create Java neural network components. The model consisting of a multilayer perceptron neural network identifies simultaneous leakages in multiple regions successfully. When the size of the input dataset increases from a set of 10 values to a set of 1500 values, the mean square error of outputs increases by 128 times. But when this change is from a set of 1500 values to a set of 12000 values, the mean square error increases by 1.6 times. Thus, the total mean square error decreases drastically with the increase in input size, leading to the conclusion that the model is stable and scalable.
\end{abstract}

Keywords: ANN (Artificial Neural Network), MLP.

Cite this Article: Pradyot Aramane, Akshay Bhattad, Madhwesh.M, Nishant Aithal, Akshay.P, Prof.Prapulla S.B and Dr.Shoba.G, Iot and Neural Network Based Multi Region and Simultaneous Leakage Detection in Pipelines, International Journal of Advanced Research in Engineering and Technology, 10(6), 2019, pp 61-68.

$\mathrm{http}: / /$ iaeme.com/Home/issue/IJARET?Volume $=10 \&$ Issue $=6$ 


\section{Pradyot Aramane, Akshay Bhattad, Madhwesh.M, Nishant Aithal, Akshay.P, Prof.Prapulla S.B and Dr.Shoba.G}

\section{INTRODUCTION}

In recent years, there has been a rising interest for water because of the rising worldwide population and urbanization. On the other hand, water infrastructure such as pipes has been deteriorating due to aging. Under these conditions, new technologies in the water infrastructure have been required to enable the distribution of high quality water to users in a safe and cost effective manner, from the perspective of efficiently using our world's precious water resources. The proposed system uses an artificial neural network to detect the leakage points in a water pipeline. ANNs have found wide application in simulating very complex relationships and as such found wide application in modelling water resources management problems including leakage detection, water distribution network optimization, water pipeline replacement and rehabilitation, water demand forecasting, and pressure monitoring. The type of neural network used in the proposed system is a multilayer perceptron neural network. The Multi-layer Perception Neural Network (MLP) is a network in which there is an input layer consisting of nodes that simply accept the input values. The outputs of neurons in a layer are inputs to neurons in the next layer. The last layer is called the output layer. Layers between the input and output layers are known as hidden layers.

\section{LITERATURE SURVEY}

On-Line Monitoring System of Water Leakage Detection in Pipe Networks with Artificial Intelligence [4]: The above mentioned paper uses the Radial basis Neural Network for modelling. It is a multilayer perceptron, which considers the median value of data and the distance between the data while setting the weight values of the neural network. The training data is obtained using the Hydraulic simulation tool. Smart Water Monitoring System Using Wireless Sensor Network at Home/Office[5]: The Smart Water monitoring System proposed in the paper uses light and water sensors to detect the height of water in the reservoir. In case of leakage detection in pipes, Pressure sensors are used. This sensor is fixed between the pipes. In case any pipes burst, it will be indicated to the agent and the underground sensor uses a force sensitive resistors (FSR) for pressure measurements in the proposed WSN for pipeline monitoring [6]. Application of SVM and ELM Methods to predict Location and Magnitude Leakage of Pipelines on Water Distribution Network [7]: This paper presents the method to predict the location of leakage and Magnitude of leakage using Support vector machines and Extreme learning Machine (ELM). The water network is modelled in EPANET and the data is retrieved for training purpose. This paper compares the above two machine learning techniques and concludes that the ELM approach is better in terms of accuracy than the SVM An approach to leak detection in pipe networks using analysis of monitored pressure values by support vector machine [8]: The mining of data obtained by collection of pressure sensors values to obtain the information about the location and size of leaks. With the help of this model the data sets are obtained which are further used for training and testing. If the training data has continuous values in the output, then SVM is implemented as a regressor so that it acts as function approximate or if the output values are in different classes then SVM is trained as Classifier. Simulation of Water Distribution Networks the Use of EPANET [9]: The simulation of water distribution networks (WDNs) using EPANET. The optimal design of water distribution networks. Minor losses caused by bends and fittings can also be accounted for by assigning the pipe a minor loss coefficient. The hydraulic head lost by water flowing in a pipe walls. Hydraulic Modelling of the Water Distribution System of Tavua/Vatukoula, Fiji [10]: A water supply distribution system comprises of a complex network of interconnected pipes, service reservoirs and pumps. The hydrological interpretation is difficult given no records of mine abstraction, and there are gaps in the hydrological flow records, which have required synthetic substitution based on the Nakauvadra catchment flow records. Just as storm sewer analysis is 
driven by the watershed runoff flow rate, water distribution system analysis is driven by customer demand. Water usage rates and patterns vary greatly from system to system and are highly dependent on climate, culture and local industry.

\subsection{Problem Definition}

The task at hand is to develop a water pipeline monitoring and maintenance support system which periodically collects data and analyses it, to detect and locate leakages and send warnings to the user. The system should be automated and should have the least possible manual interruptions required in the maintenance of the system to ensure it is efficient and fast.

\subsection{Organization of paper}

The following part of paper describes the designing of our proposed approach in section II. It describes the network architecture and subsystem structure. Section III shows the results and efficiency of proposed system and is explained while considering different scenarios. In Section IV the proposed work is concluded.

\section{PROPOSED APPROACH}

The problem was addressed by adopting a neural network paradigm. The water pipeline is treated as a set of regions which each start and end at pressure sensors. These pressure sensor readings serve as input to the system. The system consists of a recurrent multilayer perceptron neural network. This network identifies regions of the pipeline with leakages when it is fed with input pressure readings [11]. The network consists of four layers where two are hidden layers while the other two are input and output layers [12-14]. These layers are interconnected to each other in the following manner: First layer of network which is the input layer with 8 neurons and 1 bias neuron is connected to all the layers below it. The second layer (the first hidden layer) of the ANN is connected to third layer, and this third layer (the second layer) is connected to the fourth layer (the output layer) and this layer is also connected to its previous layer to form a cycle [15]. The hidden layers do not have bias neuron. The activation function chosen for the network is the sigmoid function. The learning rule for the network is back propagation with gradient descent algorithm [16-22]. This ANN is trained and tested with the extracted data (pressure values). In figure 1.1 the 8 input layer neurons represent the 8 sensors, and the 7 output layer neurons represent the 7 regions, in which the leakage may occur. The ANN takes input pressure values and gives the probability of leakage in each region as output. This output is stored as a csv file. A website is created to display the results of the processing in the form of comprehensive graphs. This website takes the csv file as input.

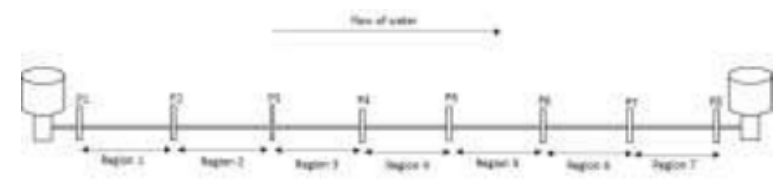

Figure 1: Pipeline region division

System Architecture

Large systems are decomposed into subsystems that provide some related set of services. The initial design process of identifying these sub-systems and establishing a framework for subsystem control and communication is called architectural design and the output of this design process is a description of the software architecture. 


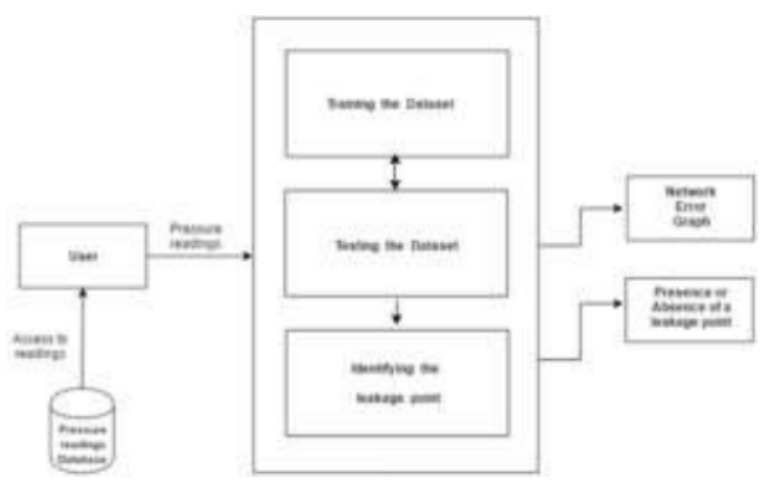

Figure 2: Overall Architecture of the System

The architectural design process is concerned with establishing a basic framework for a system. It involves identifying the major components of the system and communications between these components. The System consists of the user, who gives the access to the Pressure sensor readings dataset to the Leakage Detection model, as shown in Figure 2.1. The multilayer perceptron neural network is then trained with the training dataset. The network, is later tested with testing dataset, and presence or absence of a leakage point is found. The network also shows the Network Error graph.

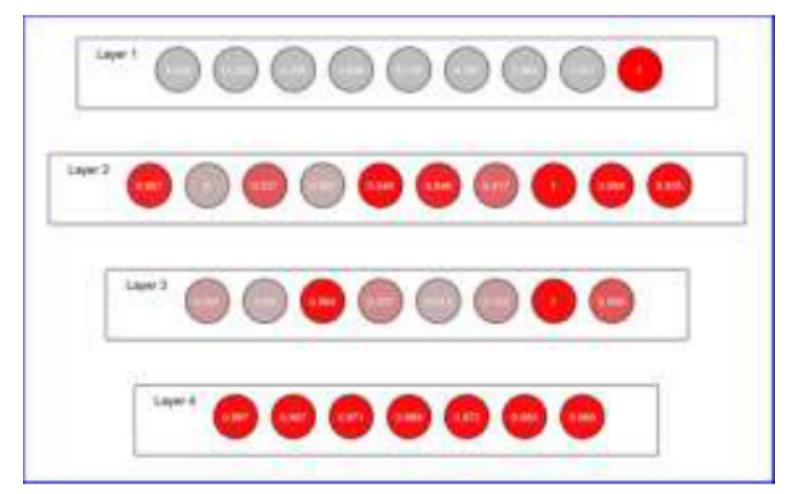

Figure 3: Multilayer perceptron neural network */

Neural Network structure: The multilayer perceptron developed has four layers. 1. Input layer: It has eight neurons receiving input from eight sensors. 2. First Hidden layer: It has ten hidden neurons which receive inputs from the input layer as well as from the next hidden layer (Back propagation). 3. Second Hidden layer: It has eight hidden neurons, which receive inputs from first hidden layer and the input layer. 4. Output layer: It has seven output neurons. The output neurons represent seven regions between the eight sensors.

\section{RESULTS AND EFFICIENCY}

The Leakage Detection System was carried out as a part of an automation system for immediately finding a leakage point in a WSN connected pipeline network. The system was successfully tested for deployment. This chapter lists the results of the experiment conducted and the inferences that were made from the testing. The evaluation metrics have been listed and the results have been accordingly listed out in this chapter. The results were obtained from a combination of the information acquired through initial process discovery as well as statistics gathered from multiple runs of the system.

The system was mainly designed to reduce human effort in a nearly deterministic process. Hence, the best metric to determine the success of the system is a comparison between the 
human and the system using various real-time reading metrics of the leakage data. The detection is taken as a metric here, and comparisons are performed based on the same.

The dataset on which the experiment was conducted was the set of sensor readings. The data was obtained over the course of different runs of the pipeline. The performance metrics have been compared with the values measured manually during the same period. The dataset used for training the network had 12000 values which were a mixture of sets containing leakage in every region and sets with leakage in multiple regions.

The leakage detection model is meant to reduce human effort in determining the leakage point and avoiding loss of water. It is multiple times faster than a human performing the same task. The convention taken in this task is, that in case of leakage the Actual Output is 1 , and 0 in case of no leakage. So, the measured expected output is either 0 or 1

Table 1: Input for different Regions

\begin{tabular}{|c|c|c|c|c|c|c|c|c|c|c|c|c|c|c|c|}
\hline $\begin{array}{l}\text { Cas } \\
\text { e } \\
\text { No }\end{array}$ & Input 1 & Input 2 & Input 3 & input 4 & Input 5 & Input 6 & input 7 & input \& & $\begin{array}{l}\text { Regi } \\
\text { on } 1\end{array}$ & $\begin{array}{l}\text { Re } \\
\text { gio } \\
\text { n } 2\end{array}$ & $\begin{array}{l}\text { Re } \\
\text { gio } \\
\text { n } 3\end{array}$ & $\begin{array}{l}\text { Re } \\
\text { gi } \\
\text { on } \\
4\end{array}$ & $\begin{array}{l}\text { Re } \\
\text { gi } \\
\text { on } \\
5\end{array}$ & $\begin{array}{l}\text { Re } \\
\text { gio } \\
\text { in } 6\end{array}$ & $\begin{array}{l}\text { Re } \\
\text { gi } \\
\text { on } \\
7\end{array}$ \\
\hline 1 & 8.092918 & 20.23341 & 7.256018 & $\begin{array}{r}6 \operatorname{scs} g 0 \\
8\end{array}$ & $\begin{array}{r}10.1794 \\
3\end{array}$ & 5791393 & $\begin{array}{r}486360 \\
3\end{array}$ & $\begin{array}{r}432676 \\
9\end{array}$ & 0 & 0 & 0 & 0 & 0 & 0 & o \\
\hline 2 & 7.985412 & 11.73341 & $7.29: 025$ & $\begin{array}{r}699675 \\
4\end{array}$ & $\begin{array}{r}11.1072 \\
2\end{array}$ & 5845745 & $\begin{array}{r}494529 \\
5\end{array}$ & 438512 & 1 & 0 & 0 & 0 & 0 & 0 & 0 \\
\hline 3 & a. 028253 & 20.23341 & 4.314359 & 695426 & $\begin{array}{r}11 . \operatorname{cet} 3 \\
7\end{array}$ & 586725 & $\begin{array}{r}494529 \\
5\end{array}$ & 438512 & 0 & 1 & 0 & 0 & 0 & 0 & o \\
\hline 4 & a. 026256 & 20.23341 & 7.314369 & 496426 & $\begin{array}{r}10.5505 \\
2\end{array}$ & 586725 & $\begin{array}{r}494529 \\
5\end{array}$ & $\begin{array}{r}4.4 C 262 \\
6\end{array}$ & 0 & 0 & 1 & 0 & 0 & 0 & o \\
\hline 5 & 7.957907 & 20.23341 & 7.256918 & $\begin{array}{r}6 \mathrm{sccs} 0 \\
\mathrm{~B}\end{array}$ & $\begin{array}{r}424 \div 42 \\
6\end{array}$ & 5.826404 & $\begin{array}{r}494529 \\
5\end{array}$ & 438512 & 0 & 0 & 0 & 1 & o & c & 0 \\
\hline
\end{tabular}

\begin{tabular}{|c|c|c|c|c|c|c|c|c|c|c|c|c|c|c|c|}
\hline 6 & 3.026258 & 2023341 & 7.314369 & 6.56425 & 1036725 & 4.29725 & 4.945255 & 4,402626 & 0 & 0 & 0 & 0 & 1 & 0 & 0 \\
\hline 7 & 8.025258 & $202334:$ & 7291028 & 6.56425 & 10.36725 & 5. 86725 & 2.945255 & $4.386 \div 2$ & 0 & 0 & 0 & 0 & 0 & 1 & 0 \\
\hline 8 & B. 026258 & $202334:$ & $7.29102 \mathrm{~B}$ & 6.56425 & 10.36725 & 5.86725 & 4.945255 & 2.326769 & 0 & 0 & 0 & 0 & 0 & 0 & 1 \\
\hline 9 & 8.025258 & 11.73341 & 7.291629 & 6.56425 & 4.241426 & 5.86725 & 2.945295 & 438612 & 1 & 0 & 0 & 1 & 0 & 1 & 0 \\
\hline 10 & 8.025268 & $112334:$ & 4.314369 & 6.56425 & 11.06637 & 4.29725 & 4.945295 & 438612 & 1 & 1 & 0 & 0 & 1 & 0 & 0 \\
\hline
\end{tabular}

The table 6.1 shows the various input values and the regions corresponding to it. It has eight inputs, as selected in the Multilayer perceptron neural network. Every two inputs have a region between them, forming seven distinct regions.

Table 2: Resultant values in the network

\begin{tabular}{|c|c|c|c|c|c|c|c|c|c|c|c|c|c|c|}
\hline $\begin{array}{l}\text { Case } \\
\text { No }\end{array}$ & AO1 & $\mathrm{AO2}$ & $\mathrm{AO} 3$ & $\mathrm{AOS}$ & AOS & AOG & $\mathrm{AOT}$ & E1 & E2 & EJ & E4 & E5 & E6 & E7 \\
\hline 10 & 1 & 1 & 0 & 0.0003 & 1 & $0 \operatorname{ceg} 2$ & 0.0374 & 0 & 0 & 0 & $-0,0003$ & 0 & .00032 & -0.0374 \\
\hline 9 & 1 & 0 & 0 & 1 & 0 & 1 & 0 & 0 & 0 & 0 & 0 & 0 & 0 & 0 \\
\hline
\end{tabular}


In Table 6.2, the Final results are shown considering a sample leakage point. The output for different regions is shown. Here AO means Actual Output of the region and E means the error in the output of the region. We can see that the actual output very closely matches the expected output. The error seen is minimal. Thus, the system gives optimal results.

The total network error graph of the network is calculated and given below in Figure 6.1. The error network graph shows that with the increase in iterations the error decreases to a particular point, where further iterations cause very less impact on error. This indicates that the model works efficiently with considerable size of dataset and efficiently determines the leakage point. Assuming the threshold is 0.5 (Output values greater than 0.5 implies Leakage). The output values which have values less than 0.5 shows that there is no leakage in that region. The network identifies leakages in each region individually. In datasets, where there is only a single point of leakage, the network successfully identifies that region. The network also identifies leakages in case of multiple regions of leakage successfully. The output shows all the regions with leakage with values above 0.5

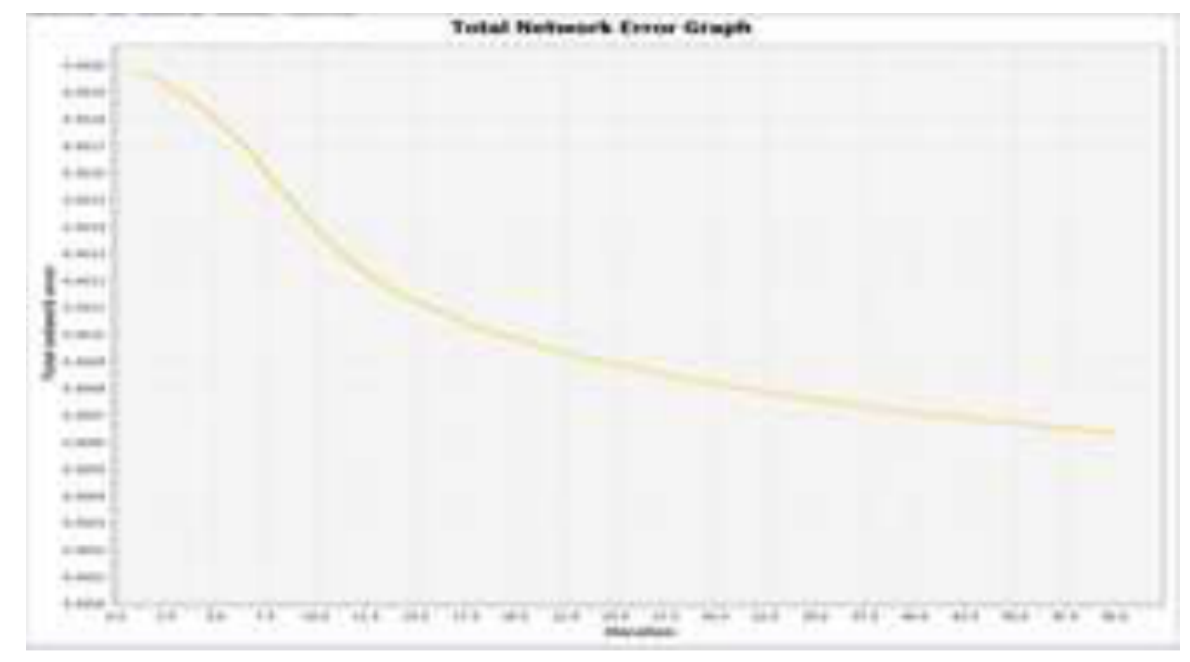

Figure 4: Total network Error graph

\section{CONCLUSION}

The neural network paradigm for the problem is currently the most effective possible and most beneficial approach. The multilayer perceptron leakage detection model has proved to be accurate and stable as the size of the data to be processed increases. Learning occurs in the perceptron by changing connection weights after each piece of data is processed, based on the amount of error in the output compared to the expected result [12]. The extraction module reads the dataset from csv files and feeds the values to the neural network. The neural network then processes this data gives marked regions as the output. The leakage detection model is capable of detecting both single and multiple regions of leakage with good accuracy. The leakage points are shown in the dashboard for comfortable and comprehensive viewing of the leakage points. The dashboard dynamically updates the set of leakage points, based on the dataset used in the network [25]. The total mean square error achieved in the output values is considerably low and the model stabilizes as the input data size increases (as the increase in error saturates). Thus, the model is efficient, reliable and scalable. 


\section{REFERENCES}

[1] UN-Water, FAO, Coping with water scarcity. Challenge of the twenty-first century, 2007

[2] UNDP, Human Development Report, beyond scarcity: Power, poverty and the global water crisis. United Nations Development Programme, New York, 2006

[3] A.Daoudi, M.Benbrahim snd K.Benjelloun, "An Intelligent System to Classify Leaks in Water Distribution Pipes", Proceedings of World Academy of Science, Engineering and Technology Volume 4 February 2005

[4] A.Ejah Umraeni Salam, , Muh.Tola, Mary Selintung and Farouk Maricar, On-Line Monitoring System of Water Leakage Detection in Pipe Networks with Artificial Intelligence, ARPN Journal of Engineering and Applied Sciences, VOL. 9, and NO. 10, OCTOBER 2014, pp 1817 -1822 .

[5] Ms T.Deepiga, Ms A.Sivasankari, Smart Water Monitoring System Using Wireless Sensor Network at Home/Office, International Research Journal of Engineering and Technology (IRJET) - Volume: 02 Issue: 04 | July-2015, pp 1305 - 1314

[6] J Goldman and D Estrin,"Distributed Sensing System for Water Quality Assessment and Management", Feb 1, 2010, pp 312-356.

[7] Application of SVM and ELM Methods to predict Location and Magnitude Leakage of Pipelines on Water Distribution Network, International Journal of Advanced Computer Research, Volume 5, June 19th 2015

[8] An approach to leak detection in pipe networks using analysis of monitored pressure values by support vector machine, Institute of Electrical and Electronics Engineers - IEEE 2009.

[9] Mohammad N. Almasri, "Simulation of Water Distribution Networks The Use of EPANET", Optimal Design of Water Distribution Networks -An-Najah National University - 2015

[10] Hydraulic Modelling of the Water Distribution System of Tavua/Vatukoula, Fiji, University of Southern Queensland Faculty of Health, Engineering and Science - 2014

[11] Eugine Makaya and Oliver Hensel, "Modelling flow dynamics in water distribution networks using artificial neural networks - A leakage detection technique". International Journal of Engineering, Science and Technology Vol. 7, No. 1, 2015

[12] K. Hornik, M. Stinchcombe and H, White, "Multilayer feed forward networks are universal approximators," Neural Networks, Vol. 2, No. 5, 1989, pp. 359-366.

[13] K. Hornik, M. Stinchcombe and H, White, "Universal approximation of an unknown mapping and its derivatives using multilayer feed forward networks," Neural Networks, Vol. 3, No. 5, 1990, pp. 551-560.

[14] G. Cybenko, "Approximation by superposition of a sigmoidal function," Mathematics of Control, Signals and Systems, Vol. 2, No. 4, 1989, pp. 303-314.

[15] Haykin, S., Neural Networks: A Comprehensive Foundation, 2nd Edn., New Jersey: PrenticeHall, 1999

[16] V. P. Plagianakos, G. D. Magoulas and M. N. Vrahatis, "Deterministic nonmonotone strategies for efficient training of multilayer perceptrons," IEEE Transactions on Neural Networks, vol. 13, no. 6, 2002, pp. 1268-1284.

[17] H. Chen, "Neural Network Training and Pruning Using Multiple Regressions," Ph.D. Dissertation, The University of Texas at Arlington, 1997.

[18] F. J. Maldonado and M. T. Manry, "Optimal pruning of feed forward neural networks based upon the Schmidt procedure," Thirty-Sixth Annual Asilomar Conference on Signals, Systems and Computers, Pacific Grove, CA, 3-6 November 2002, pp. 1024-1028,

[19] E. M. Johansson, F. U. Dowla and D. M. Goodman, "Back propagation learning for multilayer feed-forward neural networks using the conjugate gradient method," International Journal of Neural Systems, vol. 2, no. 4, pp.291-301, 1991.

[20] P. Werbos, Beyond regression: New Tools for Prediction and Analysis in the Behavioral Sciences, Ph.D. Dissertation, Committee on Applied Mathematics, Harvard University, Cambridge, MA. 1974. 
Pradyot Aramane, Akshay Bhattad, Madhwesh.M, Nishant Aithal, Akshay.P, Prof.Prapulla S.B and Dr.Shoba.G

[21] Gaurav Bothra, Sweta Bhattacharya and Deepa P, A Study of Handwritten Characters Recognition Through Neural Networks Using Data Mining Approaches, International Journal of Mechanical Engineering and Technology, 9(10), 2018, pp. 870-878.

[22] D. B. Parker, "Learning logic," Invention Report S81-64, File 1, Office of Technology Licensing, Stanford University, 1982.

[23] Yerremsetty Tayar, R.Siva Ram Prasad and S.Satayanarayana, An Accurate Classification of Imbalanced Streaming Data Using Deep Convolutional Neural Network, International Journal of Mechanical Engineering and Technology, 9(3), 2018, pp. 770-783.

[24] D. E. Rumelhart, G. E. Hinton and R. J. Williams, "Learning internal representations by error propagation," in D. E. Rumelhart and J. L. McClelland (Eds.), Parallel Distributed Processing, Vol. I, Cambridge, MA, the MIT Press, 1986.

[25] Jaykumar Jagani and Prof. Kamlesh Patel, an Enhanced Algorithm for Classification of Web Data for Web Usage Mining Using Supervised Neural Network Algorithms, International Journal of Computer Engineering and Technology (IJCET), Volume 5, Issue 4, April (2014), pp. $48-56$

[26] C. Mahesh and E. Kannan, An Intelligent System with A Novel Approach for Diagnosing Hepatitis Viruses using Generalized Regression Artificial Neural Network, International Journal of Civil Engineering and Technology, 8(9), 2017, pp. 898-910.

[27] H. H. Chen, M. T. Manry and H. Chandrasekaran, "A neural network training algorithm utilizing multiple sets of linear equations," Neuro computing, vol. 25, no. 1-3, 1999, pp. 55-72.

[28] Ruby Singh and Dr. Niraj Singhal, an Optimized Vehicle Parking Mechanism Using Artificial Neural Network, International Journal of Computer Engineering and Technology, 10(01), 2019, pp. (102)-(109).

[29] Dharmendra Kumar singh , Pragya Patel, Anjali Karsh and Dr.A.S.Zadgaonkar, Analysis of Generated Harmonics Due to Cfl Load on Power System Using Artificial Neural Network, International Journal of Electrical Engineering and Technology (IJEET), Volume 5, Issue 3, March (2014), pp. 56-68

[30] J. Vijayashree, J. Jayashree. An Expert System for the Diagnosis of Diabetic Patients using Deep Neural Networks and Recursive Feature Elimination. International Journal of Civil Engineering and Technology, 8(12), 2017, pp. 633-641.

[31] C. Yu and M. T. Manry, "A modified hidden weight optimization algorithm for feed forward neural networks," Thirty-Sixth Annual Asilomar Conference on Signals, Systems and Computers, Pacific Grove, CA, 3-6 November 2002, , pp. 1034-1038.

[32] Ch. Mamatha, P. Buddha Reddy, M.A. Ranjit Kumar and Shrawan Kumar, Analysis of Big Data with Neural Network. International Journal of Civil Engineering and Technology, 8(12), 2017, pp. 211-215.

[33] Garima Tripathi and Jagruti Save, an Image Processing and Neural Network Based Approach for Detection and Classification of Plant Leaf Diseases, International Journal of Computer Engineering and Technology (IJCET), Volume 6, Issue 4, April (2015), pp. 14-20

[34] Yong Z, Yanpeng C, Peng J, Hoogkee J, Development of a web-based decision support system for supporting integrated water resources management in Daegu city, South Korea. Expert system with Applications. 39(11), 2012, pp 10091-10102.

[35] Dharmendra Kumar singh, Ekta Singh Thakur, Smriti Kesharwani, Dr. A.S.Zadgaonkar , Analysis of Generated Harmonics Due to Single Phase Pwm Ac Drives Load on Power System Using Artificial Neural Network, International Journal of Advanced Research in Engineering and Technology (IJARET), Volume 5, Issue 2, February (2014), pp. 173-185

[36] Dharmendra Kumar singh Dr.Moushmi Kar Dr.A.S.Zadgaonkar, Analysis of Generated Harmonics Due to Transformer Load on Power System Using Artificial Neural Network, International Journal of Electrical Engineering and Technology (IJEET), Volume 4, Issue 1, January- February (2013), pp. 81-90

[37] M.Venkatesan and Dr.S.Selvakumar Raja, An Implementation of Invanet Hybrid Architecture and Hybrid Routing Protocol Using Deep Neural Network, International Journal of Mechanical Engineering and 2018, pp. 377-386. 